\title{
Les films d'animation numérique et la répétition du cinéma
}

Thomas Lamarre

\section{(2) OpenEdition}

Journals

Édition électronique

URL : http://journals.openedition.org/transtexts/85

DOI : 10.4000/transtexts.85

ISSN : 2105-2549

Éditeur

Gregory B. Lee

Édition imprimée

Date de publication : 1 janvier 2007

Pagination : 59-84

ISSN : 1771-2084

\section{Référence électronique}

Thomas Lamarre, "Les films d'animation numérique et la répétition du cinéma », Transtext(e)s

Transcultures 跨文本跨文化 [En ligne], 2 | 2007, mis en ligne le 11 juin 2009, consulté le 04 mai 2019.

URL : http://journals.openedition.org/transtexts/85 ; DOI : 10.4000/transtexts.85 


\title{
Les films d'animation numérique et la répétition du cinéma
}

\author{
THOMAS LAMARRE
}

\begin{abstract}
This paper explores the ways in which the theories and production of digital media can entail certain assumptions about the history of cinema. Particularly important is an exploration of two very different ways of conceptualizing and actualizing the repetition of cinema in digital animation. On the one hand, in Lev Manovich's Language of New Media and in Sakaguchi Hironobu's 2001 digitally animated film Final Fantasy: The Spirits Within, one finds a form of 'fatal repetition,' which insists on mediatic closure and systemization and reinforces a teleological approach to history. On the other hand, in recent theories of early cinema and in Rintarô's 2001 animated film Metropolis, one sees a form of 'serial repetition,' in which repetition of cinema results in the production of incommensurable temporalities that defy resolution or completion while promising redemption. Between these two tendencies new possibilities arise for thinking between cinema and digital animation.
\end{abstract}

\section{Introduction}

L'étude des nouveaux médias semble appeler une théorie de la répétition historique. Les débats entourant les nouveaux médias ont commencé, avec un regain d'insistance ces dernières années, à faire référence à l'émergence du cinéma afin d'évoquer une époque où le cinéma lui-même en était un. Ceci est particulièrement vrai des études sur le cinéma numérique, les films d'animation numérique et les jeux vidéo. Parfois, les critiques ne font que souligner l'incroyable nouveauté apportée par les nouveaux médias, et offrent des descriptions et des interprétations plus ou moins détaillées de ces prétendues nouveautés tout en s'opposant aux « vieux» médias tels que l'imprimé ou le film. Mais d'ordinaire, il n'est cependant pas suffisant de célébrer la nouveauté de ces nouveaux médias : insister sur la nouveauté par opposition aux vieux médias revient à se confronter à un problème historique. Après tout, les vieux médias ont autrefois été nouveaux. Et parfois, les vieux médias sont réactualisés - les disques vinyls peuvent faire un retour, avec une conception différente de leur matérialité, être manipulés de façon particulière, arrêtés, scratchés, sautés ; comme Christian Marclay qui, au milieu des années 1980, sablait, craquait, brisait et réparait des disques afin de trouver de nouveaux sons. Ainsi, même lorsqu'on ne cherche qu'à énumérer ou caractériser ce qui est nouveau à propos des nouveaux médias, on est confronté au problème de la répétition historique - sans parler du problème de l'ancien réactualisé et $\mathrm{du}$ nouveau obsolète. 
Philip Rosen a certainement raison de voir dans le discours sur la nouveauté des nouveaux médias un écho du problème de la modernité. Il tente de prouver que l'opposition habituelle entre le numérique et ses contreparties (que ce soit l'analogique ou l'indexical), a tendance à devenir une lutte du nouveau contre l'ancien. Pour ce faire, il évoque le débat soulevé par Reinhart Koselleck sur la problématique de l'historicité moderne, qui relève précisément de la répétition. ${ }^{1}$ La notion de nouveauté, explique Koselleck (via Nietzsche), suppose un paradoxe temporel particulier - un paradoxe dont le sous-titre pourrait être « est-ce que la rupture est une forme de relation?» Une fois que la rupture devient la valeur dominante pour comprendre l'histoire (le nouveau abolit l'ancien), ce qui est nouveau aujourd'hui est ancien le lendemain. Les modernes se lancent dans une recherche frénétique de la dernière nouveauté, de la nouveauté la plus neuve. Le résultat est une culture du renouveau perpétuel, à l'intérieur de laquelle, paradoxalement, rien ne change. L'innovation constante inhibe la transformation au lieu de la permettre. ${ }^{2}$ Néanmoins, refuser la rupture de la même façon - refuser, par exemple, qu'il y a quelque chose de nouveau à propos des nouveaux médias, c'est aussi paralyser les forces de transformation, c'est les rejeter catégoriquement. Le problème est de penser la rupture en tant que relation - ou plus spécifiquement, la manière selon laquelle la rupture fait apparaître une série de relations comme par magie.

Le postmodernisme a déjà réinventé, déplacé et intensifié ce paradoxe (ainsi l'idée de Lyotard selon laquelle le postmodernisme est, en réalité, un moment de naissance innovante au sein de la modernité), et requis aussi une plus grande attention à la problématique de l'émergence. Malgré tout, le paradoxe persiste et Rosen suggère que, dans les débats sur les nouveaux médias, la tendance à définir l'indexical ou l'analogique comme antagoniste au numérique est une tentative pour dépasser et nier ce paradoxe. ${ }^{3}$ Finalement, parce qu'il a tendance à associer l'indexicalité avec l'histoire et l'historicité, Rosen souhaite attribuer une certaine forme d'indexicalité aux nouveaux médias afin de prouver leur historicité. Pour lui, nier tout degré d'indexicalité au numérique revient à refuser toute relation au réel et donc à exclure toute potentialité à l'histoire. Bien que nous soyons d'accord avec le constat de Rosen sur l'existence d'un paradoxe temporel au cœur de la problématique des nouveaux médias, nous doutons de la nécessité de mettre en place un moment fondamental (l'index, l'analogue, ou un contact avec le réel) afin de penser de manière temporelle et historique. Son analyse soulève donc des questions importantes. Est-ce que l'on a besoin d'un discours sur les origines pour penser le mouvement et la transformation? Un discours moderne ou moderniste est-il la meilleure manière de comprendre l'innovation ou la transformation?

Cet article aborde le problème de la répétition. Dans les deux premières sections, nous nous pencherons sur les nouveaux médias en relation avec l'histoire du cinéma (c'est-à-dire, le prétendu cinéma indexical, analogique, fondé sur la photographie). Nous nous intéressons premièrement à une des pistes de réflexion portant sur la relation entre les nouveaux médias et le cinéma dans The Language of New Media par Lev Manovich. Il ne s'agit cependant pas de réduire la richesse de son propos à une simple formule. Mon propos consiste plutôt à montrer comment l'insistance sur la nouveauté historique des nouveaux médias est souvent fondée et garantie par l'idée d'une clôture historique du cinéma. Considérer le cinéma analogique comme un système clos et cohérent a comme conséquence que le cinéma peut servir de fondation et d'origine non reconnues au cinéma digital. Ceci nous mène à ce que nous appellerons une répétition fatale - l'idée de l'inévitabilité de certaines formes de clôture et de systématisation, de la fin comme à la fois complétion et destruction historique. La

\footnotetext{
${ }^{1}$ Philip Rosen, Change Mummified: Cinema, Historicity, Theory, University of Minnesota Press, 2001, p.303-304.

${ }^{2}$ Reinhart Koselleck, Futures Past: On the Semantics of Historical Time, MIT Press, 1985.

${ }^{3}$ Rosen, Change Mummified, p. 314.
} 
deuxième section analysera le film de Sakaguchi, Final Fantasy: The Spirits Within (2001) comme exemple de répétition fatale.

La deuxième partie de cet article se tourne vers les études récentes sur le film à ses débuts, qui nous donnent une image très différente du cinéma passé et présent. Ces études accentuent la force ou le potentiel du film, présentant le cinéma comme divergent à ses origines. Elles mettent donc en place un modèle différent pour penser la relation entre les anciens et les nouveaux médias. Une autre forme de répétition apparaît ici, qui n'est pas fondée sur la répétition de tendances systématiques implicites dans la forme, mais plutôt dans une tendance de potentialisation complice de la forme que nous nommerons répétition sérielle. Elle est cruciale pour comprendre l'impact des nouveaux médias sur les films d'animation et les séries cultes produits pour un public de masse, auquel nous nous intéressons particulièrement. La dernière section utilise Metropolis (2001), film d'animation réalisé par Rin Tarô, afin d'y puiser une autre façon d'imaginer la relation entre le cinéma et l'animation numérique.

\section{La systématisation des formes filmiques}

The Language of New Media de Lev Manovich fait allusion au problème de la répétition historique lorsqu'il remarque que les nouveaux médias n'ont pas encore produit leur version de The Birth of a Nation. Cela revient à dire que les nouveaux médias n'ont pas encore créé $\mathrm{d}$ 'objet fondateur qui pourrait consolider un langage propre analogue au langage cinématographique établi. ${ }^{4}$ Il suggère donc la possibilité que les nouveaux médias soient limités d'une certaine manière, voire destinés, à répéter l'histoire du cinéma.

Le point de vue de Manovich sur l'histoire du cinéma évoque quelque chose proche de la tragédie. Le cinéma, en tant que révolution au sein des médias, a inévitablement produit The Birth of a Nation - contre lequel l'avant-garde a perpétué les possibilités révolutionnaires du film (A Man with a Movie Camera). Peut-être est-ce moins une tragédie qu'une fatalité. L'histoire du cinéma exprime la fatalité des formes, une systématisation inévitable qui ruine la promesse du cinéma alors même qu'il dépeint le futur à travers les failles que l'avant-garde trouve dans le présent.

Des lignées vénérables d'études cinématographiques ont étayé cette interprétation de l'histoire du cinéma comme révolution manquée. Le film fatal se décline en de nombreuses versions. Noël Burch remet en question une vision selon laquelle les caractéristiques même de la caméra détermineraient l'échec de l'expression cinématographique. Il s'interroge sur les travaux de :

«Marcelin Pleynet, Jean-Louis Baudry et d'autres [qui] décrètent que les propriétés optiques de l'objectif photographique (et, donc cinématographique), cette technologie monoculaire tout droit issue de l'idéologie bourgeoise, étaient comme un "péché originel » du septième art, une fatalité historique inhérente à sa nature, et ne pouvant être libérées par des pratiques subversives. » 5

Ces critiques considéraient que les propriétés des lentilles, de l'appareil photographique et cinématographique, retranchaient inévitablement le cinéma dans des formes spécifiques de représentation. Cependant, Burch conçoit une autre version de la fatalité du cinéma. Il avance, au contraire, qu'il n'y avait rien d'inévitable concernant l'essor de certains modes de représentations de l'espace et du volume; bien qu'aujourd'hui ce mode puisse paraître

${ }^{4}$ Manovich, The Language of New Media.

${ }^{5}$ Noël Burch, La Lucarne de l'infini: Naissance du langage cinématographique, Nathan, 1991, p.155. 
hégémonique, ce n'était pas une conséquence inévitable des propriétés de l'appareil cinématographique. Burch parle "d'un mode institutionnalisé de représentation », pour souligner le fait que le mode hégémonique de représentation cinématographique est le produit de pressions institutionnelles, qui sont elles-mêmes liées à des préoccupations financières (le capitalisme). En d'autres mots, les préoccupations socio-économiques «conditionnent» la direction prise par la représentation cinématographique. La lentille monoculaire n'a pas simplement contraint le cinéma à reproduire l'idéologie bourgeoise. Malgré tout, nous faisons face à une révolution manquée, sans doute un peu moins fatale, puisque Burch recherche des alternatives aux origines du mode institutionnalisé de représentation.

David Bordwell, Janet Staiger et Kristin Thompson offrent une autre vision, moins polémique, moins désespérée, une vision en apparence neutre et empirique de la fatalité $d u$ cinéma dans la systématisation : la mise en place d'un "style cinématographique classique ", l'ultime film fatal. Contrairement à Burch, ils ne mettent pas l'accent sur la manière dont les préoccupations socio-économiques conditionnent l'expression filmique, mais reconnaissent les développements historiques parallèles. Selon la description de Miriam Hansen :

«[ils] conçoivent le cinéma classique comme système intégral et cohérent, un système qui met en relation un mode spécifique de production (fondé sur des principes fordistes d'organisation industrielle) et une série de normes stylistiques interdépendantes, élaborées autour de 1917 et demeurées en place plus ou moins jusqu'en 1960.» ${ }^{6}$

Il en résulte le style hollywoodien classique qui est structuré :

«par des motivations et par une cohérence des causalités, du temps et de l'espace; par une clarté et une redondance qui guide les opérations mentales du spectateur; par des séries formelles de répétitions, de variations, de rime, d'équilibre et de symétrie ; et en général par la composition unitaire et bien délimitée.» 7

Dans ces discours sur l'émergence d'un langage, d'un code, d'un style ou d'un mode de représentation cinématographique standardisé, le cinéma semble condamné à l'échec, à revenir sur sa promesse, à saboter sa propre révolution ou, du moins, à être forcé d'accepter cette inévitable systématisation et cette standardisation. Ces histoires qui décrivent et problématisent la standardisation de l'expression cinématographique soutiennent l'idée d'un cinéma clos et complet qui, à son tour, hante et habilite les débats sur les nouveaux médias. Manovich, par exemple, prend pour acquis que le cinéma est devenu un système (un langage, un code, ou un style) complet, cohérent, essentiellement clos et intelligible. Apparaît alors la question de la répétition historique. Est-ce que le cinéma numérique est condamné à répéter le cinéma, à devenir un système clos, à créer son propre Birth of a Nation?

\section{Animer la fin du cinéma}

Final Fantasy: The Spirit Within (2001), un film inspiré d'une série extrêmement populaire de jeux vidéo, semble avoir été conçu afin de remplir les critères du «cinéma numérique par excellence », annoncé par Manovich deux ans auparavant dans son article "What is Digital Cinema? » (1999). Il écrit :

«En principe, avec suffisamment de temps et d'argent, on peut créer ce que sera le cinéma numérique par excellence : quatre-vingt dix minutes remplies par 129600 images entièrement peintes à la main à partir de rien, mais qui sont impossibles à distinguer de la

\footnotetext{
${ }^{6}$ Miriam Bratu Hansen, "The Mass Production of the Senses : Classical Cinema as Vernacular Modernism", in Reinventing Film Studies, sous la direction de Christine Gledhill et Linda Willams, Arnold, 2000, p.336.

${ }^{7}$ Hansen, 336.
} 
photographie traditionnelle; ${ }^{8}$

De la même façon, les créateurs de Final Fantasy insistent :

«Aucun modèle de référence n'a été utilisé, aucun transfert numérique à partir d'être humain réel n'a été fait afin de créer ces personnages; ils ont tous été construits à partir de rien depuis l'ordinateur. ${ }^{9}$

Dans les deux cas, le cinéma numérique par excellence suppose la reproduction de l'action telle qu'elle se présente dans la réalité (live-action), fondée sur la photographie ou sur le cinéma analogue.

La relation entre le cinéma et l'animation numériques reste difficile à comprendre. Tant de termes semblent caractériser cette relation. Cette abondance de termes indique une certaine perplexité. Est-ce de la reproduction, de la simulation, de l'incorporation, de la recréation, de l'inspiration, de l'évocation, de la transformation, de la sublation?

Il est essentiel pour Manovich de distinguer les nouveaux médias des anciens par rapport à leur relation au réel. Ce qui est important est que le cinéma, parce qu'il est indexical, entre en contact et saisit le réel. A peu de choses près, son interprétation de la relation entre analogue et numérique correspond à l'avis général, tel que le résume Rosen :

«Tandis que l'inscription analogique est relativement continue et dépend d'un contact physique entre différentes substances (la peinture de l'artiste sur une toile, par exemple), l'inscription numérique est relativement discontinue et dépend d'un code en apparence arbitraire d'éléments rationnels et discrets (les chiffres). » 10

Mais alors, que saisissent les nouveaux médias, si ce n'est le réel? Les nouveaux médias saisissent les autres médias. Ils sont fondamentalement omnivores, ce qui est selon Manovich, une partie de leur problème.

Une question fondamentale sur les médias et la médiation est ici soulevée. ${ }^{11}$ Si un média peut incorporer ou subsumer tous les autres, peut-il aussi servir de médiateur à l'ensemble de leurs relations? Quel genre de synthèse est-ce, si toutefois cela en est une? Le résultat est-il simplement une machine géante, en pleine expansion, qui écrase tout sur son passage - à la manière de ces conglomérats éclectiques qui apparaissent si souvent à la fin de nombreuses productions de Otomo Katsuhiro (tel que Akira ou Roujin Z), qui implosent au ralenti en révélant leurs pièces détachées ? Ou encore, les nouveaux médias sont-ils capables d'être les médiateurs de tous ces éléments hétérogènes? Les nouveaux médias sont-ils par excellence la machine hégélienne de l'omni-médiation? Ces questions à propos des médias et de la médiation méritent une attention particulière.

Pour ce qui est du cinéma numérique, c'est un exemple bien particulier parmi les nouveaux médias dont le but est de saisir le cinéma. Donc, comme l'animation, il est éloigné d'un cran (au moins) du contact avec le réel. Son but est bien de saisir le cinéma, de le reproduire ou de

\footnotetext{
8 Lev Manovich, “What is Digital Cinema”, in The Digital Dialectic: New Essays on New Media, MIT Press, p.173-192. Le chapitre 6 de The Language of New Media, "What is Cinema", reprend le même argument, en mettant l'accent sur «l'art de l'index».

${ }^{9}$ Cette idée est considérée suffisamment importante pour l'inclure dans les remarques liminaires du DVD qui sont, par ailleurs, assez brèves.

${ }^{10}$ Rosen, Change Mummified, p.302.

${ }^{11}$ Dans Remediation : Understanding New Media (MIT Press, 1999), Jay Bolter et Richard Grusin suggèrent que le média peut être défini comme «ce qui remédie» (p.19). Cependant, parce qu'on ne sait pas si l'on doit comprendre la médiation au sens classique du terme, leur étude soulève un certain nombre de questions posées ici, essentiellement, est-ce que les médias médiatisent au sens classique du terme?
} 
le simuler. C'est précisément le but de Final Fantasy. Son objectif est de faire un cinéma «à partir de rien ", c'est-à-dire sans le média analogique qui définit le cinéma. Le but est de faire « un cinéma sans cinéma » réalisé à partir « d'un cinéma à travers l'animation ». Mais, si l'on saisit le cinéma indexical, est-ce que l'on reproduit aussi son contact avec le réel? Est-il amputé ou transformé, ou est-ce qu'on s'en est éloigné? Est-ce vraiment de la médiation?

Il est révélateur de noter que les spectateurs attentifs de Final Fantasy ont tout de suite réalisé que la fabrication des acteurs de toutes pièces à partir d'un ordinateur, telle que leurs créateurs l'avaient prétendue, était trompeuse. Le problème réside dans l'utilisation par les réalisateurs de capteurs de mouvements. Comme nous le rappelle le texte de la jaquette du DVD, la capture de mouvement fut effectuée grâce à « un membre de l'équipe de production en costume moulant noir bordé de 37 marqueurs qui simulait des mouvements réels, captés par 16 caméras spéciales reliées à des écrans d'ordinateur. » Les programmeurs utilisaient ensuite ces données pour créer des squelettes en trois dimensions qui servaient de base pour le design et l'animation des acteurs numériques. En d'autres termes, comme beaucoup de spectateurs le firent remarquer sur des critiques en ligne, il ne s'agissait pas du cinéma numérique par excellence ; des caméras avaient été utilisées. Bien que les cinéastes n'eussent pas filmé de véritables acteurs par des moyens photographiques, ils avaient saisi les mouvements de véritables personnes afin de mieux créer des acteurs qui bougeaient avec réalisme, c'est-à-dire de façon à respecter les conventions cinématographiques. Final Fantasy avait donc trompé ceux qui avaient considéré l'expression «à partir de rien» de façon littérale.

On pourrait prétendre que la capture de mouvement est tout simplement un bon exemple de la tendance qu'ont les nouveaux médias à utiliser la photographie et des éléments de liveaction comme un média parmi tant d'autres. En d'autres termes, le numérique peut utiliser l'analogique, mais seulement comme un composant numérisé parmi tant d'autres. Cependant, si certaines personnes qui ont vu Final Fantasy ont parlé de tromperie, c'est parce que la saisie analogique du réel - même sous la forme d'une capture de mouvement - n'est pas seulement un autre média numérisé parmi d'autres. C'est en quelque sorte une condition fondamentale de ce cinéma numérique par excellence. L'insistance des cinéastes sur le fait qu'aucun véritable acteur n'avait été utilisé ne fait qu'augmenter notre méfiance.

Un problème similaire hante l'éloignement par rapport à l'indexical. Les médias indexicaux revêtent toutes les caractéristiques fondatrices - une relation stable au réel, fondée sur leur contact avec celui-ci, qui offre des points de référence stables et aussi une identité et une histoire. Les nouveaux médias, par contre, ne touchent pas au réel et ne le mettent pas en images. Les médias numériques génèrent plutôt des images à partir de chiffres, comme distanciés de l'emprise du réel. De plus, puisque n'importe quel ancien média peut être numérisé, les nouveaux médias desserrent la prise que les anciens médias ont sur le réel, en les ouvrant aux manipulations et aux transformations qui jettent le doute sur leur emprise sur la réalité. Les médias numériques génèrent des réalités au lieu de les enregistrer. En fait, les nouveaux médias semblent menacer la logique des origines.

Cependant, Manovich, au moment précis où le lecteur pense que les nouveaux médias pourraient nous mener par-delà la logique des origines ou la remettre profondément en cause, la photographie et le cinéma live-action viennent à la rescousse. Les médias indexicaux demeurent afin de fournir la preuve qu'il y a un réel quelque part, qui peut être touché et saisi - même si les nouveaux médias ne lui sont pas directement reliés. En d'autres termes, l'indexical continue à fournir un fondement à cette version des nouveaux médias - dans une version du réalisme qui nie la capacité des médias indexicaux à construire quelque réalité que 
ce soit. C'est en ce sens que le langage des nouveaux médias est tributaire de notre capacité à imaginer la fin du cinéma. Cependant, ceci signifie la fin dans les deux sens du terme. Cette fin est à la fois la disparition du cinéma et son achèvement ou son apogée.

Final Fantasy suppose un mouvement presque identique à celui dont parle Manovich. A partir d'un grand nombre de registres différents, le film prétend pouvoir nous mener par-delà la logique des origines. Premièrement, en plus d'éviter le besoin d'endroits réels comme lieux de tournage, le film déstabilise les relations avec un lieu de production. Ce film a été produit partout et nulle part, des bribes d'information ont été transmises électroniquement entre des ordinateurs situés à différents endroits au Japon et aux États-Unis. Deuxièmement, les conventions narratives utilisées par les créateurs semblent puiser à la fois dans les jeux vidéos, les films d'Hollywood et les animations japonaises. Il semble que Sakaguchi, qui avait déjà développé l'histoire pour les jeux vidéo de la série Final Fantasy, ait travaillé avec deux scénaristes américains, Al Reinhart et Jeff Vintar, afin de donner une teinte hollywoodienne à son histoire «japonaise». En effet, en réponse à ceux qui ont perçu cette histoire comme essentiellement japonaise (et donc difficile à comprendre pour les Occidentaux), Sakaguchi avance qu'elle a été écrite à Hollywood. Bien sûr, des doutes subsistent quant à la nature supposée des conventions narratives japonaises et de leur capacité à s'affranchir de l'hégémonie hollywoodienne et des conventions cinématographiques internationales (et du risque qu'elles deviennent incompréhensibles). Mais, l'argument selon lequel l'histoire ellemême a été produite dans différents lieux à travers le monde, demeure - sans point de repère, lieu, ou source unique. Troisièmement, le nom de l'héroïne du film, Aki Ross, suggère qu'elle est à la fois japonaise et américaine. Bien sûr, d'autres éléments pourraient fournir $\mathrm{d}^{\prime}$ avantage d'information sur ses origines, mais dans le film, la nature des origines n'importe pas autant que la capacité d'Aki Ross à avoir plusieurs origines. Durant le film, par exemple, elle porte en elle un extraterrestre qui est emprisonné à l'intérieur d'un champ de force situé dans sa poitrine, ce qui lui permet d'ouvrir sa conscience de l'espace au-delà de la terre, sur une autre planète. C'est donc à travers ces trois éléments que le film remet en question la logique des origines en démultipliant ses endroits de production et ses origines.

Quatrièmement, et ceci est d'autant plus important, ce film raconte l'histoire d'une extinction violente et du bouleversement d'une origine. Dans ce film, une planète rouge est détruite après un événement qui n'est pas sans rappeler un holocauste nucléaire, cependant, ces habitants, déportés sur la Terre (la planète bleue) par l'explosion, survivent sous une forme spectrale. Ces fantômes, qui se nourrissent de l'esprit des humains, en viennent à menacer de détruire aussi la vie sur Terre. De façon assez littérale, le film envisage la fin d'une planète à la fois comme une annihilation et un achèvement. Les anciens habitants de la planète rouge, autrefois en guerre, forment dans la mort une chaîne de vie fantomatique; il forme un tout unitaire, bien que malveillant - un vestige de vie spectrale fondu en un à l'échelle planétaire, chaque créature étant liée aux autres de façon inquiétante. Parce qu'elles ne sont plus apparemment en guerre les unes contre les autres, les créatures chassent maintenant les hommes. Le film essaye de montrer comment la Terre peut atteindre une certaine unité, elle aussi - une unité bienveillante et régénératrice - qui sera en opposition avec le danger spectral que représentent les fantômes. Évidemment, plusieurs humains devront mourir tout au long du film avant que la Terre puisse atteindre sa belle unité sur Gaïa. À la fin, l'issue de la bataille entre la vie verte et bleue et les spectres rouges dépendra de la victoire de la guérison sur la destruction, de l'empathie sur la colère, de l'amour sur la haine. Et c'est seulement parce que Aki Ross vit avec un organisme extraterrestre à l'intérieur de son corps, tout près de son cœur, qu'elle comprendra le sort tragique des envahisseurs et qu'elle pourra résoudre le conflit entre les deux peuples tel que nous venons de le décrire. 
Afin d'assurer que la réalisation finale de l'unité sous-jacente à la vie sur Terre ne soit pas perçue autrement que comme bienveillante, de nombreux adversaires sont structurés autour $\mathrm{d}^{\prime}$ archétypes fascistes et militaristes qui, les yeux remplis de haine et le visage défait par leurs pulsions violentes, proposent $\mathrm{d}$ 'attaquer leur propre planète dans le but $\mathrm{d}$ 'annihiler les envahisseurs fantômes. La fin proposée par les méchants est tout simplement une fin, une destruction totale. La fin proposée par les gentils est, quant à elle, une apogée de la vie. Cela rend possible la conception d'une bonne et d'une mauvaise mort - une mort mythique, créatrice, qui permet de racheter les fautes, contre une mort militaire, destructrice, qui conduit à l'annihilation. La bonne fin demande elle aussi la mort, mais d'un type tout à fait différent : le sacrifice de soi pour les autres. Le héros (Grey) doit se sacrifier pour assurer que l'unité de la vie puisse régner. C'est seulement avec la perte du bien-aimé, ou plutôt son incorporation à l'esprit de Gaïa, que l'amour atteint ses objectifs. La logique sous-jacente du film étant une logique de fin, il y a néanmoins beaucoup plus de mort que de vie.

Au final, Final Fantasy raconte l'histoire de la vie qui perd violemment ses origines, mais qui survit malgré tout. Du point de vue des envahisseurs fantômes, c'est une histoire d'origines absentes ou, plus précisément, d'origines traumatisantes. Leur terre natale n'a pas tout simplement disparu, elle a été perdue de façon traumatisante. Dans le film, la terre natale des humains est continuellement sous la menace de disparaître. Ce discours de la perte suggère une logique psychanalytique - à l'intérieur de laquelle la crainte de la perte devient plus traumatisante que la perte elle-même - dont la reconnaissance pourrait servir à faire apparaître le processus de reniement implicite dans la répétition mélancolique de la perte. Malgré tout Final Fantasy est plus proche de la thérapie « New Age » que de la psychanalyse. En définitive, il y a une guérison et une rédemption plutôt qu'un déplacement, des signes de refoulement et des masques. La fin est une fin, sous la forme de la rédemption et du salut de la Terre. Plus précisément, le film imagine la rédemption de la terre natale, d'une origine, mais à l'échelle mondiale.

L'histoire de Final Fantasy répète donc le problème de la fin qui est implicite dans la notion même de production d'un cinéma numérique qui $\mathrm{n}^{\prime} \mathrm{a}$ pas recours au média cinématographique - un cinéma réel fait à partir de rien, c'est-à-dire un réel sans origine. La narration fait écho à la production, au sens où les deux sont structurées autour de la création d'un monde - pas seulement le monde du film (ses lieux, ses acteurs, ses actions), mais aussi le monde filmique (sa réalité) - à partir de rien. Tout le cinéma devra être généré sans le cinéma. Autrement dit, la forme cinématographique doit être générée en l'absence de son média, de sa matérialité. C'est comme si une matrice originaire avait été perdue. Il en résulte un nouveau média emprisonné dans une quête paniquée de ses origines.

Dans le film, cette quête des origines (pour l'esprit qui se cache à l'intérieur) attire notre attention sur l'effet spécial répété sans cesse - celui des formes digitales humanoïdes qui, ironiquement, ont puisé leur vivacité chez un acteur réel à partir de capteurs de mouvement. En d'autres termes, l'angoisse des origines nous oblige à revenir dans ce lieu étrange où le corps numérique puise son mouvement, sa vie, d'un véritable humain. C'est alors le problème du réel à l'intérieur du cinéma numérique qui transforme l'histoire de Final Fantasy en une quête pour des signes de vie - une recherche désespérée pour Gaïa.

L'intérêt de Final Fantasy se situe dans sa capacité à explorer de façon concrète le problème du réel cinématographique. Le projet qui consiste à « faire du cinéma sans cinéma » dépend de la fin du cinéma. L'impasse à laquelle est confronté ce projet peut être expliquée facilement: pour que le cinéma numérique entre en relation avec le réel, il doit échouer. Si ce film avait produit une simulation numérique vraiment parfaite, personne ne le considérerait différent 
du cinéma traditionnel. En d'autres termes, le cinéma numérique doit répéter le cinéma dans la différence. Ce projet demande donc une double perspective dans laquelle nous verrions du cinéma sans voir le cinéma - d'où les analyses du film à partir du concept d'étrangeté (Livia Monnet) ou de rotoscoping (Mark Langer). ${ }^{12}$ Le problème de Final Fantasy est qu'il ne semble jamais vraiment capable de savoir comment évoquer sa différence vis-à-vis du cinéma, ou du moins de situer cette double perspective, dans le but de transmettre ainsi la signification de l'importance de la différence numérique. Le film donne l'impression globale d'une tentative vaine de dépasser le cinéma.

Final Fantasy nous indique aussi une impasse importante - à laquelle les nouveaux médias vont être continuellement confrontés dans leur relation avec le cinéma, et en particulier avec ce qui relève de la production de longs métrages et de séries télévisées. De la même manière que les animations sur celluloïd et les autres formes d'animation, le cinéma numérique continuera à répéter le cinéma. (Peut-être devrions-nous appeler cette interface entre cinéma et animation: "cinémation»). L'animation digitale ou «cinémation» continuera de déplacer son attention vers la capture de mouvements, le mouvement et la vie - et donc vers des questions d'animation et de génération au lieu des questions d'enregistrement et de duplication. Et tandis que les médias numériques déplacent notre attention des questions de l'index et du réel vers celles du mouvement et de la vie (de l'animation), ils auront tendance à se fixer sur le côté humain. Le problème du traitement de l'humain devient, encore une fois, une problématique centrale. Comme le démontre Final Fantasy, voir l'homme comme une limite physique plutôt que comme un pouvoir affectif pourrait très bien résulter en une quête désespérée des origines, à l'intérieur de laquelle les anciennes identités et les vieilles histoires sont à la fois désavouées et réécrites. Dans cette perspective, Final Fantasy ne représente pas une révolution médiatique qui pourrait renverser la souveraineté cinématographique et engendrer son propre Birth of a Nation. Sa prétention à produire un nouveau monde commence et se termine plutôt avec le renouveau mythique d'un monde des plus anciens. C'est pourquoi je pense qu'il est important de rendre compte de la problématique des nouveaux médias en tant que répétition historique - il y a, après tout, différentes façons de répéter le cinéma et des façons différentes de répéter le cinéma.

\section{Réinventer la potentialité perceptuelle du cinéma}

Les appels à l'histoire qui sont au cœur de la refonte des études cinématographiques aujourd'hui, sont au mieux compris comme une tentative de modifier l'histoire du cinéma en déconstruisant l'histoire tragique du déclin du cinéma. Et c'est Deleuze qui me vient ici à l'esprit dans sa réponse à Marx. Au début du 18 Brumaire de Louis Bonaparte Marx écrit:

« Hegel fait quelque part cette remarque que tous les grands événements et personnages historiques se répètent pour ainsi dire deux fois. Il a oublié d'ajouter : la première fois comme tragédie, la seconde fois comme farce.» ${ }^{13}$

Et Deleuze répond:

«Il y a un tragique et un comique de répétition. La répétition apparaît même toujours

\footnotetext{
${ }^{12}$ Livia Monnet, dans "Invasion of the Movie Snatchers", suggère que l'effort de Final Fantasy pour construire un réel cinématographique résulte en une répétition étrange: le cinéma hante l'animation digitale (Cf. Science Fiction Studies, 31:1, Mars 2004). Mark Langer analyse Final Fantasy en fonction de la fin de l'histoire de l'animation, ce qui signifie, selon lui, la fin d'une frontière entre l'animation et le cinéma live-action; il attire l'attention sur les effets hyperréels générés par ordinateur qui ont brouillé ces frontières. Voir asifa.net/SAS/articles/. Récemment, à la conférence du SAS à Farnham (2003), il a signalé une autre forme d' « impossibilité » ou d'étrange dédoublement de l'animation : le rotoscoping. En fait, nous pourrions voir Final Fantasy comme un lieu unique de double vision dans lequel le numérique essaie de supplanter l'indexical, ce qui génère des effets étranges au sein même de son échec.

${ }^{13}$ Karl Marx, Le 18 Brumaire de Louis Bonaparte, Éditions Sociales, p.15
} 
deux fois, une fois dans le destin tragique, l'autre dans le caractère comique. » ${ }^{14}$

Une grande quantité d'études sur les premiers films et les films muets ont découvert une époque avant que le cinéma ne soit cinéma, avant que les images animées ne soient construites d'un point de vue discursif et codifié intentionellement comme une forme de divertissement différent des autres formes de spectacle tels que les spectacles de magie, le vaudeville, les entresorts, les lanternes magiques. En réalité, le cinéma a toujours été une farce, une comédie.

Un aspect crucial des études récentes sur les débuts du cinéma est la redécouverte (c'est-àdire la ré-invention) de la force du cinéma, avec un sens nouveau ou renouvelé de l'impact de disruption et de transformation du cinéma sur la perception et sur la communauté, une force oubliée dans l'importance accordée au défaut fatal et au déclin tragique du cinéma. Comme le remarque Scott McQuire :

«Là où plusieurs critiques ont accentué l'impact disjonctif du cinéma sur la perception humaine, les analyses récentes ont tendance à mettre l'accent sur le rôle du cinéma dans la production d'un spectateur-sujet unifié [...]. Là où les premiers auteurs étaient frappés par la force du déplacement cinématographique opéré par la personnification de la vision dans le cinéma, les analyses ultérieures ont mis l'accent sur la structure systématique de cette identification «primaire» comme moyen d'atteindre une forme particulière de clôture narrative. » 15

McQuire nous rappelle que les expériences et les théories du cinéma à ses débuts sont diamétralement opposées aux théories cinématographiques qui ont percé après la deuxième guerre mondiale et dominé le paysage intellectuel à partir des années 1970. Là où les critiques d'après-guerre ont commencé à considérer le cinéma comme un mécanisme du conservatisme social, comme une façon de construire et contrôler une expérience cinématographique normative, les auteurs antérieurs considéraient souvent la potentialité d'un éveil politique ou d'une subversion culturelle. En somme, le choc engendré par le cinéma promettait au départ une transformation et une révolution, mais graduellement, la naturalisation et la standardisation de la perception cinématographique ont été assimilées à des mécanismes de régulation et de contrôle.

Avec la récente ré-invention des études cinématographiques centrées sur les premières expériences cinématographiques et théories du cinéma, les commentateurs ont néanmoins réinventé la force perceptuelle du cinéma. Cette ré-invention entraîne des conséquences importantes sur notre manière de penser les nouveaux médias. En fait, comme l'ont souligné les théoriciens des débuts du cinéma, le désir de ré-inventer la force du cinéma est issu de la crise d'un type d'expérience cinématographique lié au cinéma urbain, amené par l'invention de la vidéo, du home cinema et, ensuite, des DVDs et autres médias numériques. ${ }^{16}$ Le 100e anniversaire de la naissance du cinéma en 1995 a engendré des réflexions sur sa fin. Ce n'est pas que les films ont cessé d'être produits, mais les transformations au niveau de la production, de la réception et de la distribution du cinéma (si l'on peut encore parler de cinéma) ont été tellement significatives dans les années 1980 que les théoriciens du cinéma se sont sentis obligés de repenser son identité. À la place du soi homogène et d'une identité unitaire qui sous-tend la forme classique du cinéma et les modes institutionnalisés de représentation, les théoriciens ont ré-inventé la force perceptuelle, une notion beaucoup

\footnotetext{
${ }^{14}$ Gilles Deleuze, Différence et répétition, Paris: Presses Universitaires de France, 1968, 25.

${ }^{15}$ Scott McQuire, Vision of Modernity : Representation, Memory, Time and Space in the Age of the Camera, Sage Publications, 1998, p.70

16Miriam Hansen, "Early Cinema, Late Cinema: Transformations of the Public Sphere”, Screen 34, n. 3 (Automne 1993) p.197-210.
} 
moins stable et figée du cinéma. Et, pendant que le cinéma subissait une crise d'identité, les théoriciens ont découvert qu'elle était divergente à partir de ses origines : elle n'était pas identique à elle-même. Avec l'avènement de la vidéo et des médias numériques, il ne semblait plus possible de parler de façon concluante de l'exploitation, de la domestication et de la systématisation du potentiel cinématographique. Le cinéma apparaissait partout et nulle part (ou du moins potentiellement) et pas de manière clairement systématique. Maintenant, alors que l'on télécharge des films depuis Internet et que l'on convertit ces fichiers dans des formats et des contextes de visionnements différents, comment ne pas reconnaître la force perceptuelle des nouveaux médias? Le dénouement tragique du cinéma est estompé par l'entrée en scène de cette économie bien particulière de vol, de don et de sacrifice, de piratage et d'exorcisme.

Du point de vue des premières expériences et théories du cinéma, il est possible de penser la relation entre les nouveaux médias et le cinéma d'une autre façon. Le cinéma répète à l'avance tous les nouveaux médias. La relation entre les nouveaux médias et le cinéma peut être vue comme une fête ou un rituel, comme une forme de répétition destinée à répéter ce qui ne peut être répété, le moment où toutes les relations sont connues d'avance, mais demeurent imprévisibles. Les nouveaux médias appelleraient la force divine et démoniaque du cinéma, la font sortir de son temple et l'enferment dans toutes les maisons et les lieux de travail, à chaque coin de rue et peut-être même dans toutes ces amulettes, talismans, chapelets, ces films téléchargés sur des disques de la grandeur d'un porte-clés, tout cela au nom du cinéma. Bien sûr, cette vision du cinéma présuppose une certaine liberté (ou vie) des formes cinématographiques et de leur matérialité, et cette forme est destinée à ne pas atteindre sa fin (téléologiquement) mais à se répéter (en série).

On peut avancer que cette relation de sérialité entre le cinéma et l'animation ne permet pas une histoire ou des relations historiques. Si la sérialité rituelle aime le passé, ce n'est pas sous forme d'histoire. Sa relation au passé est iconique et cosmologique (et souvent fétichiste) sans préoccupation pour une vérification dans les faits de documents et d'artéfacts. Néanmoins, la sérialité entraîne bien un mouvement et finit ainsi par produire des sujets. Sa relation avec le temps ressemble davantage à une capture de mouvements qu'à de l'indexical. Mais on peut penser que l'index n'est qu'une variation dynamique à partir de la capture de mouvements, voire l'inverse. N'importe quel mouvement au sein de cette dynamique est considéré suffisamment réel pour produire un soi (aussi petit soit-il) et donc de fonder des mouvements conscients et inconscients de subjectivité (mémoire volontaire et involontaire). En ce sens, même si la répétition sérielle a des relations différentes avec le passé de celles habituellement considérées comme historiques, elle implique des relations qui peuvent finalement s'avérer intéressantes quoiqu'inhabituelles.

\section{L'image multidialectique}

Le film d'animation Metropolis (2001) est une adaptation sur grand écran du manga Metropolis (1949) - une œuvre de jeunesse de l'artiste de manga le plus renommés de tous, Tezuka Osamu - et aussi l'évocation du film muet Metropolis de 1927.

Ce film d'animation a suscité beaucoup d'attention pour avoir rassemblé tant de talents et tant de différentes visions d'une métropole du futur, habitée par des humains et des robots. Rin Tarô, le réalisateur, célébré pour ces nombreux dessins animés adaptés de mangas (de Galaxy Express 999 de Matsumoto Reiji à X de CLAMPS) a collaboré avec Otomo Katsuhirô comme scénariste du film. Otomo est lui aussi reconnu pour ses talents d'artiste et de réalisateur de mangas, particulièrement depuis la série Akira et le long métrage qu'il en a tiré. 
Quelle a pu être la connexion entre le style de Rin Tarô et d'Otomo avec le style et la vision du monde de Tezuka? En plus de ces trois styles caractéristiques (ceux de Rin Tarô, d'Otomo et de Tezuka), le film d'animation a explicitement mis en contraste une des sources d'inspiration du manga de Tezuka : le Metropolis de Fritz Lang. En effet, l'histoire écrite par Otomo est tout autant redevable du film muet de Fritz Lang qu'elle l'est du manga de Tezuka. Toutefois, l'histoire présente aussi un grand nombre d'idées propres à Otomo. La scène finale, par exemple, est plus particulièrement marquée par son style que par celui de Tezuka ou de Lang.

En somme, le film d'animation Metropolis a au moins quatre sources d'inspiration distinctes qui y sont délibérément évoquées tant au niveau de la narration que dans le style. Cependant, et malgré l'hommage constant à Tezuka, aucun style ou aucune vision ne semble médiatiser les autres. En conséquence, le film risque de ne devenir qu'une mosaïque de citations et de styles, sans autre motivation apparente qu'une refonte rentable des styles de grands maîtres consacrés. De ce point de vue, l'animation Metropolis peut aussi bien être interprétée comme simplement une autre tentative de l'impulsion récente de raconter des histoires à travers différents médias.

Par exemple, le film d'animation Blood: The Last Vampire (2000) n'est qu'un chapitre dans l'histoire du dernier vampire nommé Saya (qui est aussi tueur de vampires), une histoire développée à travers trois romans, un manga, un jeu vidéo, tous situés à des périodes historiques différentes. De la même façon, The Matrix Reloaded est apparu en même temps que Animatrix (2003), une anthologie de courts métrages d'animation produits par des écrivains, des réalisateurs et des studios différents qui présente différents points de vue sur le monde de la Matrice; il y a même eu un jeu vidéo, Enter the Matrix (2003). On peut de façon légitime associer cette nouvelle tendance à la volonté de capitaliser un film avec des novélisations, des produits dérivés, des jouets, qui sont devenus le centre de la rentabilité de l'industrie cinématographique. Les jeux vidéos constituent dorénavant une source majeure de bénéfices pour un film, et parfois même la source première de bénéfices. Cette tendance à raconter des histoires à travers plusieurs médias n'est d'ailleurs pas incompatible avec l'utilisation, aujourd'hui ô combien familière, des films et des séries télévisées pour vendre des jouets. La série Bionicle (2003) de Lego est le meilleur exemple récent de jouets destinés à l'adaptation vidéo.

Malgré tout, il y a quelque chose d'intéressant au sujet de cette tendance à raconter comme une série d'histoires à travers plusieurs médias. Cette forme de sérialisation ne nécessite plus que la narration soit localisée dans un seul élément ou dans un seul média. La narration est plutôt dispersée à travers différents médias et est souvent élaborée par différents auteurs, avec des styles différents - souvent d'emblée. Chaque média constitue un niveau différent qui ajoute quelque chose à l'ensemble de l'histoire. Structurellement, ceci rappelle le mouvement entre les différents niveaux de certains jeux vidéo. Par contre, dans ces séries «intermédiatiques", les niveaux ne sont pas même vaguement hiérarchisés ni motivés en fonction d'un dénouement ou d'une résolution finale. Il y va plutôt de différentes dimensions d'un puzzle, d'un problème ou d'une quête. Mais, ce n'est peut-être pas sans ressembler à certains jeux vidéos. Blood apparaît ici comme le précurseur de la série Matrix, et va encore plus loin. Parce que Blood s'est défait (ou a tenté de se défaire) du contrôle de la vision des auteurs en laissant aux différents artistes une grande marge de manœuvre dans l'élaboration de leurs chapitres, tout en maintenant des référents historiques, ou du moins les références à des événements phares tels que la Révolution française, Shanghai dans les années 1930, les manifestations étudiantes des années 1960, et ainsi de suite. Le résultat est une production sérielle de l'histoire ou une sérialisation de l'histoire. Ces séries posent ainsi des questions 
importantes au sujet de ce qu'est l'histoire, et de l'impact des médias sur l'historiographie.

L'évocation de styles distincts et de médias différents dans l'animation Metropolis est une variation de cette nouvelle tendance à la sérialisation historique. C'est une variation qui construit ses couches et ses niveaux à partir d'œuvres antérieures élaborées sur d'autres supports. À ce niveau, sa logique rappelle celle de la citation. Mais surtout, aux côtés de citations stylistiques ou génériques, le film cite spécifiquement différentes sources en tant que médias. Ce qui est particulièrement intéressant au sujet de Metropolis c'est qu'il cherche ensuite à apporter une cohérence à tous ces styles différents en les organisant autour du problème $\mathrm{du}$ média. On se rend compte graduellement que le film est structuré autour d'une tension entre l'animation numérique et l'animation sur celluloïd, personnifiée par le robot Tima. Le film se dégage donc de la logique narrative de médiation (historique) pour mieux se plonger dans une logique de co-existence (médiatique). Le film évoque donc une rédemption plutôt qu'une synthèse ou une résolution. Ceci requiert quelques explications.

Quelques unes des images les plus saisissantes du film sont celles où le robot Tima, tel un ange, est baigné par un halo de lumière. Elle nous apparaît pâle et rayonnante, avec une crinière blonde et des yeux d'un bleu limpide, sur fond des traits sévères de la Ziggurat imposante de Métropolis. Le désign rectiligne de la Ziggurat est en contraste net avec l'éclat doux et harmonieux de Tima. La majorité du matériel promotionnel du film mettait l'accent sur ce contraste. L'image de Tima avec la Ziggurat en arrière-plan apparaît, par exemple, sur la jaquette d'une réédition du manga de Tezuka, et la couverture du livre promotionnel du film pousse le contraste un peu plus loin, avec un dessin de profil de la tête de Tima, le visage doux, angélique, rayonnant, tandis que l'intérieur de son crâne dévoile des courroies, des roues et autres mécanismes. C'est une représentation astucieuse, bien calculée de la problématique centrale du film : Tima est un robot qui ressemble entièrement à un humain tout en trahissant une nature inhumaine. D'une part, elle est une enfant, belle et innocente, angélique, voire humainement extraordinaire. D'autre part, il y a un autre aspect de Tima, son côté « caché », sombre, qui découle de sa nature mécanique.

Les dernières séquences du film nous montrent le sombre destin de Tima, lorsque son soidisant "père » (son concepteur Duke Red) la prépare à sièger sur le trône au sommet de la Ziggurat. Il avait demandé que Tima soit conçue à l'image de sa fille décédée tant aimée, mais afin de l'aider à se rendre maître de la cité et du monde, de la métropole mondiale (selon la structure générale du film, la métropole figure comme représentation métonymique du monde). Une suite d'événements permet au robot d'échapper au contrôle de son "père » pour se réfugier dans la métropole où elle tente d'échapper à ses poursuivants avec l'aide d'un garçon nommé Kenichi, qui ne sait pas qu'elle est un robot. Durant la scène finale, après que son père l'a capturée, le contraste entre les deux Tima jaillit et la part mécanique et démoniaque transperce la peau de la Tima éclatante et angélique. La machinerie envahit son corps, découvrant ses mécanismes internes, laissant seulement quelques morceaux de son corps angélique. Toute une suite d'images montrent son visage mi-ange mi-machine. Même dans les ultimes scènes de destruction, pendant que la Ziggurat tombe, alors que la démoniaque Tima tente d'attraper Kenichi, on continue de voir des bribes de l'angélique Tima. Et les créateurs du film n'ont apparemment pas pu s'empêcher de recourir au cliché : juste avant que le robot ne se précipite dans la mort, il redevient pour un court instant l'angélique Tima et reconnaît son ami Kenichi. Malgré tout, la force de film réside dans sa représentation de Tima en tant qu'être presque littéralement déchiré entre deux possibilités, deux identités, deux natures, et deux mondes.

Le contraste entre les deux Tima sert à organiser et à structurer le film entier. C'est aussi le 
lieu où les différentes sources d'inspiration du film se rencontrent. Le contraste entre les deux Tima permet à Otomo, le scénariste, d'évoquer ou de ressusciter des images familières aux amateurs de ses projets précédents (qui sont des icônes d'Otomo) : celles d'une machine omnivore, mais néanmoins autopoétique qui rappellent Akira, Roujin Z et Spriggan. En même temps, le contraste permet de garder l'esprit du manga de Tezuka, dans lequelle le robot Michi incarne la tension entre le démoniaque et l'angélique, entre le mal et le bien, entre les adultes et les enfants, entre la domination et l'innocence. Cependant, contrairement au robot Michi du manga de Tezuka qui change son identité sexuelle de garçon en fille et de fille en garçon, le robot Tima est définitivement une fille. À cet égard, Tima s'inscrit dans la tradition du robot Futura du Metropolis de Fritz Lang. ${ }^{17}$ En fait, le contraste entre les deux Tima permet à trois différentes histoires de robot de coexister: histoires distinctes mais apparemment sans contradiction.

Il est important de se rappeler que dans le film de Lang, le mauvais père (Johan Frederson) construit Futura à l'image de la Vierge Marie, dans le but d'anéantir l'esprit des travailleurs à qui elle donne l'espoir et le désir d'un avenir meilleur. Le film d'animation Metropolis repose clairement sur l'histoire d'une exploitation injustifiée de Lang (et de la romancière Thea von Habou), dans laquelle le conflit entre la tête et les mains (entre les penseurs et les travailleurs) est résolu par le cœur, ce qui devient très clair à partir de l'histoire d'amour entre Maria et le fils (Freder Frederson). L'animation Metropolis évoque la révolte des travailleurs contre l'exploitation comme dans le film de Lang, conjointement avec l'histoire de Tezuka de la révolte des robots contre la discrimination ainsi qu'avec le scénario d'Otomo des machines prenant vie et dont on perd le contrôle. Encore une fois, ces histoires coexistent d'une certaine manière sans contradiction, bien que certains spectateurs puissent penser que l'histoire n'est pas toujours très claire.

Dans le film d'animation, le cœur ou l'innocence promettent de guérir toute la gamme de problèmes évoqués par ces différentes histoires, c'est-à-dire, l'exploitation et la discrimination le complexe militaire industriel. Le «cœur » peut être interprété comme une sorte d'universel qui permet aux différentes histoires de robot de se combiner harmonieusement. Malgré tout, même si Metropolis appelle en premier lieu les humains à apprendre à comprendre, à apprécier et à aimer les robots, il montre tellement de relations différentes aux robots et à la technologie que le spectateur se retrouve finalement perplexe. Au lieu d'un seul cœur, le film en possède plutôt plusieurs, existant en quelque sorte tous à travers la double nature de Tima. C'est dans cette perspective que l'on pourrait dire de l'animation Metropolis qu'elle a surdéterminé le robot Tima. ${ }^{18}$

Par contre, l'animation Metropolis semble étrangement calculée, autant au niveau du style que de la narration, pour exposer cette surdétermination, pour la porter à la surface. Le film s'attarde sur l'impossible détermination du robot, qui demeure plein de potentiel tout en étant "sousdéterminé », presque indéterminé. ${ }^{19}$ Dans le film de Lang, par exemple, il est toujours possible de faire la distinction entre la vraie femme Maria et le robot Futura. Dans l'animation Métropolis, par contre, même si les deux personnalités de Tima sont distinctes,

\footnotetext{
${ }^{17} \mathrm{~L}$ 'évocation de robot de Lang trouve sa justification dans la postface écrite pour la première édition du manga dans laquelle Tezuka explique que, même s'il n'avait pas vu le Métropolis de Lang, il avait vu des photographies tirées apparemment de la scène du film où le robot Futura devient vivant.

${ }^{18}$ Voir l'analyse de Louis Althusser sur la surdétermination et la contradiction dans Pour Marx, F. Maspero, 1997.

${ }^{19} \mathrm{~J}^{\prime}$ ai emprunté le terme de « sousdétermination » (« underdetermination ») au texte de Paul Dumouchel sur Gilbert Simondon, in "Simondon's Plea for a Philosophy of Technology", in Technology and Politics of Knowledge, Indiana University Press, 1995, p. 225-271. J'aime le terme de « sousdétermination » en ce qu'il implique une proximité avec celui d'indétermination sans prétendre être la même chose.
} 
elles sont inséparables. D'ailleurs, le film n'offre pas de transition psychologiquement justifiée entre le robot innocent en quête d'amour et le robot qui détruira la ville. Les deux personnalités coexistent en un seul être, ce qui permet à Tima de catalyser différentes actions sans en devenir intégralement une part. Ce n'est pas étonnant que la phrase qu'elle répétera sans cesse tout au long du film est « watakushi dare » ou « qui suis-je? ». Tima est un être sans origine ou sans identité. Ou plutôt, c'est parce qu'elle possède plusieurs origines que la question des origines est soulevée.

Le problème des origines multiples est assez différent de celui de la surdétermination puisqu'il ne demande pas la médiatisation des contradictions. Par exemple, Metropolis ne présente pas ces différents scénarios comme contradictoires et ainsi ne demande pas de médiation entre des visions conflictuelles. Il situe plutôt le potentiel du robot comme une sorte de "sousdétermination", c'est-à-dire, comme quelque chose qui ne détermine pas activement, tout en n'étant pas complètement passif, quelque chose comme un média en tant que conditions matérielles.

Le problème de Tima, ses origines multiples, est essentiellement un problème de médias. Il répond à la situation énoncée par Manovich dans laquelle les différents médias sont numérisés et combinés. Bien que la numérisation dût être le grand égalisateur qui les médiatise tous, les différences entre les médias demeurent visibles, palpables, sensibles. Ces traces indiquent la sousdétermination et non pas la médiation. Elles sont des traces d'un potentiel matériel, qui est différent de l'index tel qu'il est habituellement décrit. (Je suggère plus bas que la couleur est une façon d'imaginer cette trace et de prendre en considération son aspect différentiel.) Dans beaucoup de productions des nouveaux médias, l'idée principale n'est pas d'aplanir les différences entre les médias. Le but est plutôt de maintenir l'impression d'un jeu différentiel entre les médias ou les différentes matérialités, même si ces matérialités sont déjà transformées en une seule matérialité numérique - les nouveaux médias en tant que multimédia. L'expérience en est souvent celle de médias multiples, d'origines multiples ou de matérialités multiples. La problématique ici n'est cependant pas celle du manque d'origines, d'absence de réel ou d'identité telles que les théories de l'index le sous-entendent parfois. La problématique des nouveaux médias est celle des origines multiples. Encore une fois, c'est précisément la problématique de Tima, le robot. ${ }^{20}$

Comme il arrive souvent avec la multiplicité, la multiplicité de Tima prend la forme d'une asymétrie productive (elle peut ainsi sembler binaire). Dans Métropolis, c'est le contraste entre l'innocente, angélique Tima et la Tima mécanique, démoniaque qui maintient la présence d'origines multiples. De façon significative, ce contraste est rendu par un contraste entre deux formes d'animation - l'animation sur celluloïd et l'animation numérique. Par exemple, le plan de l'angélique Tima devant la Ziggurat marque un contraste entre la dimension numérique créée par l'architecture de la ville générée par un ordinateur et les personnages aplatis, apparemment en deux dimensions, qui évoquent les surfaces peintes à la main, caractéristique principale de l'animation sur celluloïd (même si elles sont fabriquées ou coloriées à l'ordinateur). En général, l'angélique Tima évoque les techniques d'animation sur celluloïd (indépendamment de l'amélioration numérique de ses couleurs et de son modèle), tandis que les techniques d'animation numérique sont utilisées pour construire la métropole comme espace impressionnant et hiérarchisé. Par la suite, durant les séquences de fin, la Tima démoniaque émerge sous la forme de l'animation numérique. Les courroies et les roues de

\footnotetext{
${ }^{20}$ Les questions de genre sont un problème pour Tima aussi bien que pour Aki Ross. Il est crucial de remarquer que l'association entre la féminité et la matérialité fait de la femme ou des personnages féminins le lieu par excellence pour imaginer la matérialité des médias. Si cela perpétue ou non le dénigrement du corps féminin est une question qui mérite davantage d'attention dans le contexte des nouveaux médias.
} 
son substrat mécanique apparaissent sous une forme clairement numérique. Les images promotionnelles d'une Tima scindée en deux combinent astucieusement les techniques d'animation sur celluloïd et numérique. En d'autres mots, les origines multiples de Tima impliquent une asymétrie productive entre deux médias. L'ensemble du film est structuré autour de la question de leur interaction.

Harmoniser l'animation sur celluloïd et l'animation numérique est devenu un problème technique commun en animation, proche sous maints aspects de la «composition » (ce qu'on appelle en anglais compositing) du cinéma « analogue » live-action avec l'animation numérique. Comme d'autres artisans du cinéma, l'équipe de Metropolis a naturellement eu recours à un grand nombre de techniques pour mieux harmoniser ou « composer » ces différentes couches, pour camoufler l'écart entre les différentes dimensions évoquées par le style sur celluloïd et le style analogue. Cependant, et cela peut être considéré comme étant la contribution de Rin Tarô, le film souligne souvent ce contraste. La différence entre les deux médias demeurent visible, palpable, et ce délibérément, comme si la tension incarnée par Tima s'était diffuse dans le monde du film. Ou peut-être apparait-elle comme la cristallisation de cette tension? D'une façon ou d'une autre, ce film représente une expérience de médias multiples, condensés sous la forme d'une asymétrie productive, établie entre l'animation sur celluloïd et numérique, de la même façon que les deux Tima concentrent les différentes sources de l'histoire. Le résultat n'est pas sans rappeler l'expérience que Benjamin nomme l'image dialectique - une expérience de la coexistence de temporalités ou de spatialités incommensurables qui défient la résolution dialectique ou la médiatisation. Plutôt que de produire des contradictions et une médiation qui catalysent certaines formes d'actions politiques et de mouvements historiques, l'image dialectique a le grand mérite de brouiller la reproduction culturelle de la sublation historique. Cependant, elle risque d'enlever au temps historique et à la narration leur habilité à structurer les événements. Sa grande promesse s'apparente à la venue d'un temps messianique de la rédemption.

A la place de la médiation, l'animation Metropolis propose quelque chose de proche de la rédemption. C'est comme si, étant confronté à une expérience de la multiplicité qui défie la médiation, le film ne peut que s'efforcer de racheter son asymétrie structurelle. Au niveau des médias, la rédemption prend la forme de l'utilisation des couleurs. Cela revient à dire, qu'au lieu d'harmoniser parfaitement les couches de celluloïd et les couches numériques en réglant le problème de la dimension, les artisans de Metropolis ont opté pour la couleur. D'une part, ils ont utilisé la couleur pour amenuiser la disjonction entre les couches de celluloïd et les couches numériques, en peignant à la main les architectures numériques et en colorant numériquement les personnages. D'autre part, avec les programmes numériques, ils ont laissé les couleurs se développer dans deux directions incompatibles. Par exemple, ils ont peint des scènes entières de rouge et de vert éclatants, rendant une combinaison de contraste extrêmement violent avec des variations extrêmement subtiles (une infinité de tons de rouge et de vert). En d'autres termes, tandis que les couleurs semblent apporter un certain niveau de médiation (sous la forme de compositing), sa fonction première est de répéter l'asymétrie inhérente à l'expérience des médias multiples, sous la forme de super-contrastes et de variations infinies. Parfois, le film ne peut être regardé que si l'on prend en considération les couleurs, leurs croisements divisant sans besoin de s'arrêter sur les contradictions ou les médiatisations. Les couleurs font naître des profondeurs sans dimension, dans lesquelles deux, trois ou quatre couches peuvent coexister. Cela fait référence à une autre sorte de temps ou de profondeur qui promet de racheter la coexistence troublante de multiples médias, des origines, des identités, des mondes et des histoires multiples.

Mais, pour revenir à la question qui hante Rosen, est-ce qu'il est réellement possible de parler 
d'histoire ou de relations historiques? Au lieu d'une relation entre l'ancien et le nouveau, entre le passé et le présent, l'animation Metropolis suggère que, au cœur des nouveaux médias il y a une non-relation entre le vieux et le nouveau. La question nous mène précisément vers la non-contradiction et la non-médiation, vers la répétition comme (non)relation. Les nouveaux médias ne sont pas de bon augure pour l'histoire, comme façon de valider des documents fondés sur des traces indexicales du passé qui sont déterminantes (si ce n'est qu'en dernière instance). Les nouveaux médias se dirigent tout de même vers un passé qui est potentiellement moins passif, un passé qui ne se satisfait pas de jouer le rôle de fondation dans le but de maintenir les priorités temporelles pour les actions présentes. Au lieu d'une répétition fatale de la mort des systèmes antérieurs, la répétition historique engendrée par les nouveaux médias implique une répétition sérielle et s'ouvre au festival, au rituel et au mythe. Bien sûr, les spectateurs et les artisans du cinéma peuvent être forcés d'assumer une destinée tragique selon laquelle le nouveau n'est véritablement nouveau que pour autant qu'il n'ait jamais été vieux. 